\section{Efecto de un modelo de apoyo telefónico en el auto-manejo y control metabólico de la Diabetes tipo 2, en un Centro de Atención Primaria, Santiago, Chile}

\author{
ILTA LANGE $^{\text {la }}$, SOLANGE CAMPOS ${ }^{1 \mathrm{~b}}$, MILA URRUTIA ${ }^{\text {la }}$, \\ CLAUDIA BUSTAMANTE ${ }^{\mathrm{lb}}$, CLAUDIA ALCAYAGA $^{\text {la }}$, ÁLVARO TELLEZ ${ }^{2}$, \\ J. CAROLA PÉREZ ${ }^{3 \mathrm{c}}$, LUIS VILLARROEL ${ }^{4 \mathrm{~d}}$, GASTÓN CHAMORRO ${ }^{5}$, \\ ANNETTE O`CONNOR ${ }^{6 a}$, JOHN PIETTE ${ }^{7 e}$
}

\section{Effect of a tele-care model on self-management and metabolic control among patients with type 2 diabetes in primary care centers in Santiago, Chile}

Background: Telephone based self-management support may improve the metabolic control of patients with type 2 (DM2) diabetes if it is coordinated with primary care centers, if telephone protocols and clinical guidelines are used and if it is provided by nurses trained in motivational interviewing. Aim: To assess the efficacy of a tele-care self-management support model (ATAS) on metabolic control of patients with DM2 attending primary care centers in a low income area in Santiago, Chile. Material and Methods: Two primary care centers were randomly assigned to continue with usual care (control group, CG) or to receive additionally 6 telecare self-management support interventions (IG) during a 15 month period. Glycosylated hemoglobin (HbA1c) was used to measure metabolic control of DM2; the "Summary of Diabetes Self-care Activities Measure" and the "Spanish Diabetes Self-efficacy" scale were used to measure self-management and self efficacy, respectively. Changes in the use of health services were also evaluated. Results: The IG maintained its HbAlc level (baseline and final levels of $8.3 \pm 2.3 \%$ and $8.5 \pm 2.2 \%$ respectively) whereas it deteriorated in the $C G$ (baseline and final levels of $7.4 \pm 2.3$ and $8.8 \pm 2.3 \%$ respectively, $p<0.001$ ). The perception of self-efficacy in the IG improved while remaining unchanged in the $C G$ $(p<0.001)$. Adherence to medication, physical activity and foot care did not change in either group. In the IG, compliance to clinic visits increased while emergency care visits decreased. Conclusions: The ATAS intervention, in low income primary care centers, significantly increased the probability of stabilizing the metabolic control of patients with DM2 and improved their use of health services.

(Rev Med Chile 2010; 138: 729-737).

Key words: Diabetes mellitus, type 2; Primary health care; Self care; Telenursing.
'Escuela de Enfermería, Centro Colaborador en Atención Primaria OMS-OPS, Facultad de Medicina, Pontificia Universidad Católica de Chile. Santiago de Chile.

${ }^{2}$ Departamento de Medicina Familiar, Facultad de Medicina, Pontificia Universidad Católica de Chile.

${ }^{3}$ Escuela de Psicología, Facultad de Ciencias Sociales, Pontificia Universidad Católica de Chile. ${ }^{4}$ Departamento de Salud

Pública, Facultad de Medicina Pontificia Universidad Católica de Chile.

${ }^{5}$ Departamento de Enfermedades Cardiovasculares, Facultad de Medicina, Pontificia Universidad Católica de Chile.

'University of Ottawa, Canada. ${ }^{7}$ Center for Quality Improvement for Complex Chronic Conditions, University of Michigan, U.S.A. ${ }^{a}$ Enfermera.

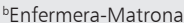

'Psicóloga.

¿Estadístico.

eSalubrista.

Fuente de apoyo financiero: Proyecto desarrollado por la Escuela de Enfermería en colaboración con el Departamento de Medicina Familiar de la Escuela de Medicina de la Pontificia Universidad Católica de Chile, financiado por FONDEF (D04i1174) (2006-2008) con el apoyo del Ministerio de Salud, Dirección de Salud, Educación y Atención de Menores de la comuna de Puente Alto, Servicio de Salud Metropolitano Sur Oriente, ENTEL Call Center S.A., Escuela de Enfermería de la Universidad de Ottawa y Programa "Quality Improvement for Complex Chronic Conditions" de la Universidad de Michigan.

Recibido el 3 de agosto de 2009 , aceptado el 8 de junio de 2010.

Correspondencia a: Ilta Lange, Escuela de Enfermería, Pontificia Universidad Católica de Chile. Fono: 3547021 , Fax: 3547025. Av. Vicuña Mackenna 4860, Santiago de Chile.

E-mail: ilange@uc.cl 
L a diabetes mellitus tipo 2 (DM2) es considerada una epidemia del siglo XXI que repercute sobre la calidad de vida del individuo y su familia, e impacta social y económicamente los servicios de salud y la sociedad en general. Su prevalencia en Chile alcanza $7,4 \%{ }^{1}$ y es significativamente mayor en adultos mayores y en grupos de más bajos recursos socioeconómicos ${ }^{2}$. Corresponde al prototipo de enfermedad crónica que exige una atención de salud estructurada, continua y centrada en apoyar al paciente en el auto-manejo de su enfermedad ${ }^{3-5}$. En este proceso se incorporan también familiares cuidadores quienes son personajes claves para este modelo de cuidados.

Se ha demostrado que el apoyo telefónico, puede contribuir a mejorar la compensación metabólica de los pacientes con DM2 ${ }^{6-8}$ si cuenta con las siguientes características: articularse con la modalidad de atención presencial otorgada en los centros de salud ${ }^{9}$, utilizar protocolos telefónicos y guías clínicas de auto-manejo basados en evidencia científica; y ser operados por enfermeras entrenadas en entrevista motivacional ${ }^{10,11}$.

Un estudio realizado en la comuna de Puente Alto, Santiago de Chile, demostró que, a pesar de los bajos ingresos de los habitantes, el 95\% de la población de diabéticos que se atiende en centros de salud públicos cuenta con teléfono y en su mayoría tiene interés en recibir apoyo telefónico periódico para el control de su enfermedad ${ }^{5}$.

Basado en estos antecedentes se diseñó, implementó y evaluó un modelo de apoyo telefónico para el auto-manejo de enfermedad crónica (ATAS) articulado con la atención de salud habitual, en centros de atención primaria públicos para pacientes con DM2 que incluyó: a) Programa de Capacitación para enfermeras de consejería telefónica y profesionales del Programa de Salud Cardiovascular (PSCV); b) Consejerías Telefónicas para pacientes con DM2, y c) Manual de Apoyo al Tele-cuidado utilizado por el paciente en su hogar, durante las consejerías telefónicas y los controles en el PSCV.

El objetivo de este trabajo fue evaluar el impacto del modelo ATAS en el control de la DM 2 en población de bajos recursos socio-económicos.

\section{Material y Método}

Este modelo fue evaluado con un diseño cuasi experimental (medición basal, 8 y 15 meses) con pacientes con DM2 que asisten al PSCV en dos centros de atención primaria de una comuna de Santiago. Estos centros fueron seleccionados por conveniencia y asignados aleatoriamente como grupo de intervención (GI) y control (GC). El estudio fue aprobado por los comités de ética de la Escuela de Enfermería, Pontificia Universidad Católica de Chile, y Servicio de Salud Metropolitano Sur Oriente.

\section{Muestra}

La población susceptible de estudio constaba de 892 pacientes en el GI y 777 pacientes en el GC. De éstos, 660 pacientes en el GI y 681 en el GC cumplían los siguientes criterios de inclusión: usuario del PSCV, tener DM2 no insulino requirente, 20 a 64 años, poseer teléfono y aceptar voluntariamente participar en el estudio. Los criterios de exclusión fueron: adicción a alcohol y drogas ilegales, enfermedad terminal, problemas psiquiátricos severos, sordera, ceguera y analfabetismo.

En la medición inicial, se reclutaron 421 pacientes en el GI y 370 en el GC; de ellos, 312 y 328 respectivamente, contaron con todos los parámetros. Al finalizar el estudio, los grupos estuvieron constituidos por 206 y 226 pacientes respectivamente (Figura 1).

\section{Procedimientos}

1. Reclutamiento: Se planificó realizarlo cuando los pacientes asistieran al PSCV, sin embargo, esta estrategia debió ser complementada con citaciones telefónicas, invitaciones escritas y visitas domiciliarias. El proceso consideró: consentimiento informado, aplicación de cuestionarios, toma de muestra de hemoglobina glicosilada, medición de presión arterial y peso. El GI recibió además el Manual de Apoyo al Tele-cuidado, con una breve capacitación para su uso. La etapa de reclutamiento duró 13 meses.

2. Intervención: Durante 15 meses, ambos grupos recibieron la atención habitual en el PSCV. Esta fue complementada, con el modelo ATAS para los pacientes del GI quienes recibieron un promedio de 6 consejerías telefónicas durante el período (rango de 1 a 13 llamadas, y una moda de 7). La duración media de las llamadas fue de 10 minutos. Las consejerías telefónicas fueron realizadas por enfermeras capacitadas en entrevista motivacional y apoyo en la toma de decisiones 


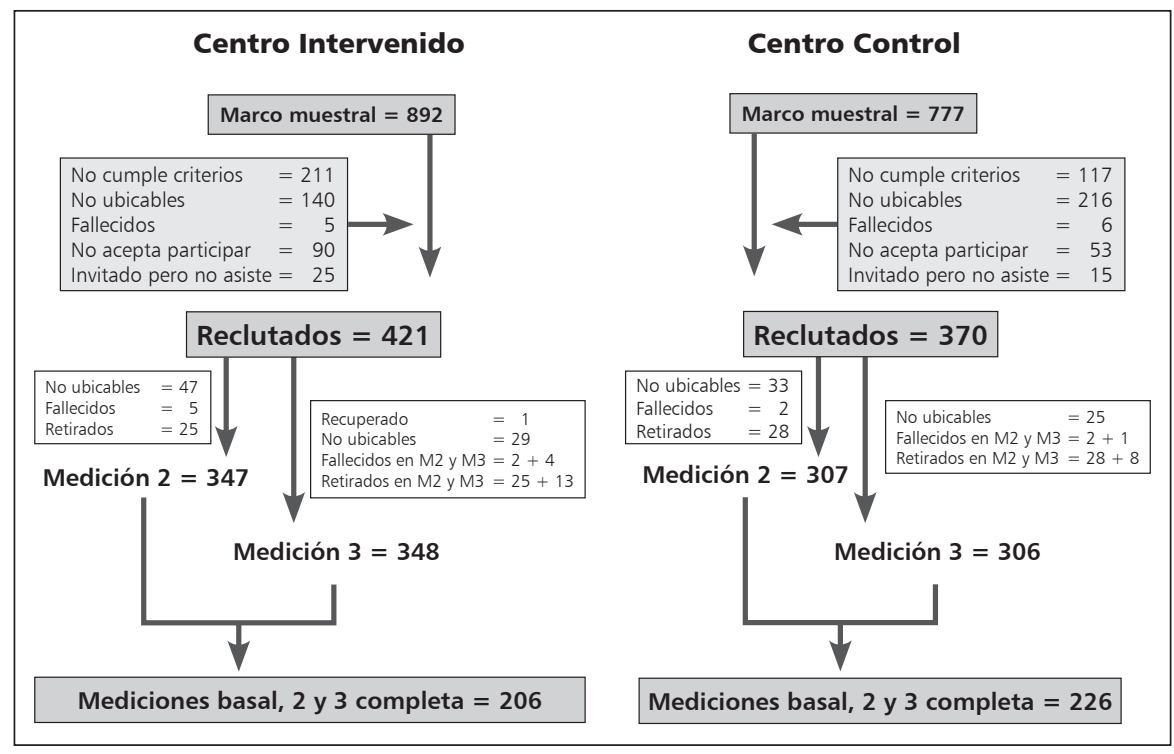

Figura 1. Constitución de la Muestra. para el auto-manejo de la diabetes. La información relevante, generada en cada intervención telefónica de apoyo al plan de cambio de conductas acordado con el paciente, se incorporaba al registro clínico electrónico (RCE) compartido con el equipo de salud presencial.

\section{Variables e Instrumentos}

Estos instrumentos son de auto-reporte:

a) Prácticas de Auto-manejo de la DM2: estas prácticas fueron medidas con ítemes basados en "The Summary of Diabetes Self-care Activities Measure"12. Incluyó:

- Adherencia a medicamentos, medida a través del número de días de la última semana que la persona ingirió "todos los medicamentos" indicados (2 ítems).

- Las prácticas de alimentación fueron diferenciadas entre aquellas saludables y no saludables. Las primeras dan cuenta del número de días en que la persona consume alimentos integrales, frutas y verduras. La segunda se refiere a ingerir alimentos ricos en grasas, golosinas y refrescos no dietéticos. La medición indica el número de días de la última semana que ingirió cada tipo de alimentos.

- Cuidado de pies, considera la revisión diaria de los pies y zapatos así como el secarse adecuadamente entre los dedos de los pies (3 ítems).
- Actividad física, considera el número de personas que realizó 30 minutos de actividad física durante 3 o más días de la última semana.

b) Percepción de Salud Social: medida a través de la escala "Spanish Social/Role Activities Limitations Scale"13. La escala mide el grado de interferencia de la enfermedad en sus actividades cotidianas (4 ítems). Un mayor puntaje (5 pts) indica que no se percibe interferencia de la DM2, en cambio un valor de 1 punto, indica que la enfermedad dificulta muchísimo su actividad. La confiabilidad es de 0,88 (Alfa Cronbach).

c) Percepción de Autoeficacia: la escala mide el grado de autoeficacia percibida para manejar su diabetes. Fue medida a través de la escala "Spanish

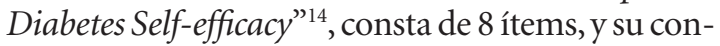
fiabilidad es de 0,79. Un mayor puntaje en la escala se traduce en un mayor sentido de autoeficacia.

\section{Utilización de Servicios de Salud}

Consideró controles en el PSCV, consultas de morbilidad en el centro de salud, atención en el Servicio de Atención Primaria de Urgencia (SAPU), consultas al Servicio de Urgencia y las hospitalizaciones. Esta información se obtuvo directamente de las instituciones de salud respectivas. 
Parámetros de Compensación Metabólica

a) Hemoglobina glicosilada (HbA1c): fue procesada en el Laboratorio Comunal.

b) Presión Arterial (PA): fue controlada según recomendación técnica del Ministerio de Salud (MINSAL). Paciente compensado presenta valores $130 / 80^{15}$.

c) Índice de Masa Corporal (IMC): el peso se controló con balanza digital y la talla se obtuvo del RCE.

\section{Estadística}

Se utilizó test "t" de Student y Análisis de Covarianza para muestras independientes para comparar promedios entre los grupos. La evolución de las variables numéricas se analizó mediante modelos lineales de efectos mixtos ${ }^{16}$. En los modelos se incluyeron variables de control, según fueran relevantes. Para las variables dicotómicas se analizó el cambio en el tiempo de la probabilidad de ocurrencia de los eventos de interés, mediante modelos logísticos de efectos mixtos. En los modelos lineales y logísticos, se analizó el efecto de interacción entre los grupos en estudio y tiempo. Se ajustaron modelos de efectos mixtos independientes para cada grupo cuando fue necesario. Se consideró significativo todo valor $\mathrm{p} \leq$ 0,05 . Para los análisis de efectos mixtos se utilizó el programa estadístico $\mathrm{R}^{17,18}$ y para los otros análisis se usó SPSS-15.

\section{Resultados}

\section{Descripción de la Muestra}

La muestra estuvo constituida mayoritariamente por mujeres, con edad promedio sobre los 50 años y una escolaridad media correspondiente a Educación Básica Completa (Tabla 1).

Tabla 1. Caracterización inicial de los grupos

\begin{tabular}{|lcc|}
\hline & $\begin{array}{c}\text { Grupo } \\
\text { Intervenido }\end{array}$ & $\begin{array}{c}\text { Grupo } \\
\text { Control }\end{array}$ \\
\hline Edad (años) & $53,76 \pm 7,7$ & $52,82 \pm 7,23$ \\
\hline Género femenino (\%) & 68,7 & 66,8 \\
\hline Escolaridad (años) & $8,1 \pm 3,26$ & $8,4 \pm 3,3$ \\
\hline Años con DM2 & $5,18 \pm 5,3$ & $5,95 \pm 6,3$ \\
\hline
\end{tabular}

\section{Efectos en Prácticas de Auto-manejo de la DM2}

Alimentación: hubo diferencias significativas en la evolución en el tiempo de las prácticas de alimentación saludable $(\mathrm{p}=0,001)$ y no saludable $(\mathrm{p}$ $=0,001$ ) entre ambos grupos (modelo ajustado). El GI disminuyó significativamente la alimentación saludable $(\mathrm{p}<0,001)$ y la no saludable $(\mathrm{p}<0,001)$, a diferencia del GC que aumentó significativamente la alimentación saludable $(\mathrm{p}<0,001)$ y mantuvo sin variación la no saludable $(\mathrm{p}=0,129)$.

Medicamentos, actividad física, cuidado de pies: no se encontraron diferencias significativas entre ambos grupos en la evolución en el tiempo, en: adherencia a medicamentos $(\mathrm{p}=0,781)$, realización de actividad física $(\mathrm{p}=0,066)$ y el auto-cuidado de pies $(\mathrm{p}=0,891)($ Tabla 2$)$.

\section{Efectos en Percepción de Salud Social y de Autoeficacia}

El modelo ajustado mostró que la percepción de salud social se mantuvo estable en el tiempo en el GI ( $p=0,968)$, mientras que en el GC mejoró ( $p$ $<0,001)$. La autoeficacia en el GI aumentó significativamente en el tiempo $(\mathrm{p}<0,001)$ y permaneció estable en el GC $(p=0,107)$ (Tabla 3$)$.

\section{Efectos en Utilización de Servicios de Salud}

El GI presentó un aumento significativo en la asistencia a controles PSCV $(\mathrm{p}<0,001)$ y esta asistencia fue significativamente más alta que en el $\mathrm{GC}(\mathrm{p}=0,002)$. El GI presentó un menor número de consultas en el Servicio de Urgencia $(p=0,041)$ respecto del GC. En cambio, no se encontraron diferencias significativas entre ambos grupos en los promedios de consultas de morbilidad ( $\mathrm{p}=$ $0,086)$, consultas en el SAPU $(p=0,097)$ y hospitalizaciones $(\mathrm{p}=0,433)$ (Tabla 4$)$.

\section{Efectos en Parámetros de Compensación Metabólica}

El nivel de HbA1c se mantuvo estable en el tiempo en el GI ( $p=0,107)$; en cambio, en el GC se observó un aumento significativo $(\mathrm{p}<0,001)$. De hecho, los valores de HbA1c al inicio del estudio fueron $8,3 \%$ en el GI y 7,4\% en el GC; a los 15 meses, el GI alcanzó 8,5\% y el GC 8,8\%.

La probabilidad de descompensación de la PA en el tiempo no difirió entre los grupos $(\mathrm{p}=0,700)$. Tampoco se observaron diferencias significativas en la evolución en el tiempo del IMC $(\mathrm{p}=0,437)$ (Tabla 5). 
Apoyo telefónico en control metabólico de Diabetes tipo 2, en atención primaria - I. Lange et al

Tabla 2. Evolución de las prácticas de auto-manejo en Salud: Datos observados medición inicial y final

\begin{tabular}{|c|c|c|c|c|c|}
\hline \multicolumn{6}{|c|}{ Medianas y Rangos Intercuartila } \\
\hline & \multicolumn{3}{|c|}{ Inicial (días/ semana) } & \multicolumn{2}{|c|}{ Final (días/ semana) } \\
\hline & G. Intervenido & & G. Control & G. Intervenido & G. Control \\
\hline Alimentación no saludable & $2(1,3-3,3)$ & & $1,7(1,3-2,7)$ & $1,7(1,3-2)$ & $1,7(1,3-2,3)$ \\
\hline Alimentación saludable & $2,5(1,5-4,5)$ & & $1,5(1,5-3,5)$ & $2(1,5-3)$ & $2(1,5-4,5)$ \\
\hline Uso de medicamentos & $7(7-7)$ & \multicolumn{2}{|c|}{$7(7-7)$} & $7(7-7)$ & $7(7-7)$ \\
\hline Actividad físicab & $43,4 \%$ & \multicolumn{2}{|c|}{$45,4 \%$} & $44,5 \%$ & $32,2 \%$ \\
\hline Cuidado de pies & $5(3,6-7)$ & \multicolumn{2}{|c|}{$5,8(4,3-7)$} & $7(5-7)$ & $7(7-7)$ \\
\hline \multicolumn{6}{|c|}{ Parámetros Modelos de Efectos Mixtosc } \\
\hline & Constante & Centro & Tiempo & $\begin{array}{l}\text { Centro* } \\
\text { Tiempo }\end{array}$ & $\begin{array}{l}\text { Variables de control } \\
\text { en el modelo }\end{array}$ \\
\hline Alimentación no saludable & $\begin{array}{c}1,060 \\
(0,068)\end{array}$ & $\begin{array}{l}-0,048 \\
(0,071)\end{array}$ & $\begin{array}{l}-0,038^{* * *} \\
(0,005)\end{array}$ & $\begin{array}{l}0,031 * * * \\
(0,007)\end{array}$ & $\begin{array}{l}\text { Alimentación no saluda- } \\
\text { ble basal }\end{array}$ \\
\hline Alimentación saludable & $\begin{array}{l}1,598 \\
(0,101)\end{array}$ & $\begin{array}{l}-0,304^{* *} \\
(0,110)\end{array}$ & $\begin{array}{l}-0,036^{* * *} \\
(0,008)\end{array}$ & $\begin{array}{l}0,063^{* * *} \\
(0,011)\end{array}$ & $\begin{array}{l}\text { Alimentación saludable } \\
\text { basal }\end{array}$ \\
\hline Uso de medicamentos & $\begin{array}{c}6,374 \\
(0,081)\end{array}$ & $\begin{array}{c}0,073 \\
(0,112)\end{array}$ & $\begin{array}{l}0,017^{* *} \\
(0,006)\end{array}$ & $\begin{array}{l}-0,0026 \\
(0,009)\end{array}$ & --- \\
\hline Actividad física & $\begin{array}{l}-1,215 \\
(0,715)\end{array}$ & $\begin{array}{c}0,194 \\
(0,208)\end{array}$ & $\begin{array}{c}0,007 \\
(0,012)\end{array}$ & $\begin{array}{l}-0,032 \\
(0,018)\end{array}$ & $\begin{array}{l}\text { Edad } \\
\text { Presencia heridas }\end{array}$ \\
\hline Cuidado de pies & $\begin{array}{c}2,544 \\
(0,119)\end{array}$ & $\begin{array}{c}0,062 \\
(0,109)\end{array}$ & $\begin{array}{l}0,065^{* * *} \\
(0,008)\end{array}$ & $\begin{array}{l}0,0015 \\
(0,011)\end{array}$ & Cuidado pies basal \\
\hline
\end{tabular}

Nota: aLas medianas y rango intercuartil se presentan en el formado $M d n$ (Primer-Tercer Cuartil). ' ${ }^{\text {L }}$ a actividad física corresponde al porcentaje de personas que realiza 3 o más días de la semana actividad física. 'Para cada variable, se presentan los parámetros de los Modelos Lineales de Efectos Mixtos estimados, con su respectivo valor, error entre paréntesis y nivel de significación estadística según: ${ }^{*} p<0,05 ;{ }^{* *} p<0,01 ;{ }^{* *} p<0,001$.

Tabla 3. Percepción de Salud Social y de Autoeficacia: Datos observados medición inicial y final

\begin{tabular}{|c|c|c|c|c|c|}
\hline \multicolumn{6}{|c|}{ Medianas y Rangos Intercuartila } \\
\hline & \multicolumn{3}{|c|}{ Inicial (puntos) } & \multicolumn{2}{|c|}{ Final (puntos) } \\
\hline & G. Intervenido & \multicolumn{2}{|c|}{ G. Control } & G. Intervenido & G. Control \\
\hline Percepción de salud social ${ }^{b}$ & $5(4-5)$ & \multicolumn{2}{|c|}{$5(3,5-5)$} & $5(4-5)$ & $5(4,75-5)$ \\
\hline Autoeficaciac & $6,75(5,25-8,38)$ & \multicolumn{2}{|c|}{$7,13(5,53-8,37)$} & $8(7,13-9)$ & $6,75(5,75-8)$ \\
\hline \multicolumn{6}{|c|}{ Parámetros Modelos de Efectos Mixtos ${ }^{d}$} \\
\hline & Constante & Centro & Tiempo & $\begin{array}{l}\text { Centro* } \\
\text { Tiempo }\end{array}$ & $\begin{array}{l}\text { Variables de control } \\
\text { en el modelo }\end{array}$ \\
\hline Percepción de salud social & $\begin{array}{c}2,201 \\
(0,118)\end{array}$ & $\begin{array}{l}-0,067 \\
(0,054)\end{array}$ & $\begin{array}{l}-0,033^{* * *} \\
(0,009)\end{array}$ & $\begin{array}{l}0,033^{* * *} \\
(0,006)\end{array}$ & $\begin{array}{l}\text { Percepción de salud } \\
\text { social basal } \\
\text { Años con diabetes }\end{array}$ \\
\hline Autoeficacia & $\begin{array}{c}6,863 \\
(0,097)\end{array}$ & $\begin{array}{c}0,075 \\
(0,134)\end{array}$ & $\begin{array}{l}0,079 * * * \\
(0,007)\end{array}$ & $\begin{array}{l}-0,091 * * * \\
(0,011)\end{array}$ & --- \\
\hline
\end{tabular}

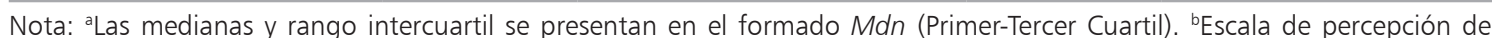
salud social (En nada/5 pts. a Muchísimo/1 punto). 'Escala de autoeficacia (mín. 1, máx. 10). dPara cada variable, se presentan los parámetros de los Modelos Lineales de Efectos Mixtos estimados, con su respectivo valor, error entre paréntesis y nivel de significación estadística según: ${ }^{*} p<0,05 ;{ }^{* *} p<0,01 ;{ }^{* *} p<0,001$. 
Tabla 4. Uso de los Servicios de Salud durante el período de intervención

\begin{tabular}{|c|c|c|}
\hline & $\begin{array}{l}\text { G. Intervenido } \\
\begin{array}{c}(n=272) \\
M \pm D E\end{array}\end{array}$ & $\begin{array}{c}\text { G. Control } \\
(n=370) \\
M \pm D E\end{array}$ \\
\hline \multicolumn{3}{|l|}{ Asistencia Programa de Salud Cardiovascular (PSCV) } \\
\hline Controles en el PSCV pre-intervención (12 meses) & $2,18 \pm 1,18^{*}$ & $1,92 \pm 1,41$ \\
\hline Controles en el PSCV intervención (12 meses) & $3,37 \pm 1,18^{* * *}$ & $2,70 \pm 1,43$ \\
\hline Delta Asistencia a Controles PSCV ${ }^{a}$ & $1,19 \pm 1,53^{* *}$ & $, 78 \pm 1,66$ \\
\hline \multicolumn{3}{|l|}{ Consultas Morbilidad y Urgencias } \\
\hline Consultas de Morbilidad en Centro de Atención Primaria & $4,03 \pm 5,52$ & $3,43 \pm 4,72$ \\
\hline Consultas en Servicio Atención Primaria de Urgencia & $1,11 \pm 1,38$ & $1,66 \pm 2,47$ \\
\hline Consultas en Servicio de Urgencias - Hospital de Referenciab & $0,34 \pm 0,86^{*}$ & $0,49 \pm 0,92$ \\
\hline Hospitalizaciones - Hospital de Referencia & $0,15 \pm 0,51$ & $0,12 \pm 0,42$ \\
\hline
\end{tabular}

Nota: aPara cada paciente, el delta se obtiene restando al número de controles que asiste durante el proceso de la intervención (12 meses), el número de controles del año anterior. 'Valores ajustados según covariable: tiempo con diabetes $(5,73$ años) y sexo. ${ }^{*} p<0,05 ;{ }^{* *} p<0,01 ;{ }^{* *} p<0,001$.

Tabla 5. Parámetros de compensación metabólica: Datos observados medición inicial y final

\begin{tabular}{|c|c|c|c|c|}
\hline \multicolumn{5}{|c|}{ Promedios y Desviación Estándar } \\
\hline & \multicolumn{2}{|c|}{ Inicial } & \multicolumn{2}{|c|}{ Final } \\
\hline & G. Intervenido & G. Control & G. Intervenido & G. Control \\
\hline $\mathrm{HbA1c}(\%)$ & $8,3 \pm 2,3$ & $7,4 \pm 2,3$ & $8,5 \pm 2,2$ & $8,8 \pm 2,3$ \\
\hline Presión arterial $^{a}$ & $36,9 \%$ & $46,4 \%$ & $48,6 \%$ & $53,6 \%$ \\
\hline IMC & $31,9 \pm 6,0$ & $31,6 \pm 6,2$ & $31,6 \pm 5,7$ & $31,1 \pm 5,7$ \\
\hline
\end{tabular}

\begin{tabular}{|c|c|c|c|c|c|}
\hline \multicolumn{6}{|c|}{ Parámetros Modelos de Efectos Mixtos ${ }^{b}$} \\
\hline & Constante & Centro & Tiempo & $\begin{array}{l}\text { Centro * } \\
\text { Tiempo }\end{array}$ & $\begin{array}{c}\text { Variables de control } \\
\text { en el modelo }\end{array}$ \\
\hline $\mathrm{HbA1c}$ & $\begin{array}{c}2,883 \\
(0,198)\end{array}$ & $\begin{array}{c}0,168 \\
(0,135)\end{array}$ & $\begin{array}{c}0,014 \\
(0,009)\end{array}$ & $\begin{array}{l}0,066^{* * *} \\
(0,012)\end{array}$ & $\begin{array}{l}\text { Hemoglobina basal } \\
\text { Años con diabetes }\end{array}$ \\
\hline Presión arterial & $\begin{array}{l}-9,834 \\
(0,737)\end{array}$ & $\begin{array}{l}-0,089 \\
(0,181)\end{array}$ & $\begin{array}{l}-0,037 \\
(0,029)\end{array}$ & $\begin{array}{c}0,007 \\
(0,018)\end{array}$ & $\begin{array}{l}\text { PA sistólica basal } \\
\text { Edad }\end{array}$ \\
\hline IMC & $\begin{array}{c}36,078 \\
(1,689)\end{array}$ & $\begin{array}{l}-0,275 \\
(0,479)\end{array}$ & $\begin{array}{l}-0,007 \\
(0,012)\end{array}$ & $\begin{array}{l}-0,014 \\
(0,018)\end{array}$ & $\begin{array}{l}\text { Sexo } \\
\text { Edad }\end{array}$ \\
\hline
\end{tabular}

Nota: aPorcentaje de pacientes con Presión Arterial compensada (valores 130/80 mmHg). IMC: índice de masa corporal. 'Para cada variable, se presentan los parámetros de los Modelos Lineales de Efectos Mixtos estimados, con su respectivo valor, error entre paréntesis y nivel de significación estadística según: ${ }^{*} p<0,05 ;{ }^{* *} p<0,01 ;{ }^{* *} p<0,001$. 


\section{Discusión}

Los resultados de este estudio muestran que el modelo ATAS fue eficaz en estabilizar la gradiente de incremento de la HbAlc esperable en pacientes con DM2, que de acuerdo a la literatura corresponde aproximadamente a un punto cada 4 años ${ }^{19}$. En cambio, en el GC se incrementó la HbAlc en más de un punto porcentual en los 15 meses. Este resultado puede predecir un mayor riesgo de complicaciones para el GC ya que el incremento de un $1 \%$ en la HbAlc, aumenta el riesgo de padecer enfermedades cardiovasculares en alrededor de un $10 \%{ }^{20}$. Así mismo, puede estimarse que la estabilización del nivel de $\mathrm{HbAlc}$ en el GI, obtenida con la aplicación del modelo ATAS implicaría 25 a 30\% de reducción en la incidencia de complicaciones micro vasculares en un período de 5 años ${ }^{21}$. De acuerdo a Khaw et al $^{22}$ un aumento de un punto porcentual en la HbAlc se asoció a 20 a $30 \%$ de aumento en la mortalidad o eventos cardiovasculares. Lo planteado por estos autores permitiría esperar que el GI presente complicaciones en forma más tardía que el GC.

El modelo ATAS mejoró la asistencia a los controles en el PSCV, donde los factores de riesgo cardiovasculares son tratados en forma simultánea. Con ello se alcanzó el número de controles recomendados por las guías clínicas del MINSAL ${ }^{23}$. Estos resultados son coherentes con la literatura que reporta la relación entre la adherencia a los controles de salud con un mejor control glicémi$\mathrm{CO}^{21,24,25}$. A pesar que los controles de morbilidad no variaron, se redujeron las consultas en el Servicio de Urgencia, lo cual es deseable desde la perspectiva de integralidad de la atención y de reducción de costos.

La adherencia al tratamiento farmacológico en personas con enfermedades crónicas alcanza en promedio el $50 \%$ y es aún menor en los países en vías de desarrollo ${ }^{26}$. En este estudio, la adherencia basal reportada por ambos grupos fue cercana al $80 \%$, cifra que puede ser exagerada, dada la reconocida tendencia del auto-reporte a sobre estimar el uso real de fármacos ${ }^{27-29}$. Dada esta condición basal, era esperable que no se demostrara diferencia en la adherencia a fármacos en ambos grupos al término del estudio.

En relación a la práctica de auto manejo para una alimentación saludable, el GC aumentó la ingesta de alimentos saludables y no modificó la ingesta de aquellos no saludables, mientras que los pacientes del GI disminuyeron significativamente ambos tipos de alimentos. Sin embargo, esta disminución no mostró efecto sobre el IMC.

La mejoría significativa de la percepción de autoeficacia en el GI es un aporte fundamental del modelo ATAS cuya meta es apoyar a los pacientes para modificar aquellas conductas que impiden el adecuado control de su enfermedad crónica. La autoeficacia es, reconocidamente, el mejor predictor de cambio de conducta ${ }^{30-32}$ y por ende, una característica que contribuye a mejorar la autonomía y la activa participación y compromiso de la persona con enfermedad crónica en el automanejo de su enfermedad.

El presente estudio tiene limitaciones que es necesario considerar. En primer lugar, la ausencia de randomización de los individuos asignados a ser controles o a recibir la intervención. Si bien era factible randomizar sujetos de un solo centro, la contaminación de la información, hubiera hecho difícil estimar la magnitud del impacto de la intervención. Por otra parte, los instrumentos de auto-reporte para medir prácticas de auto-manejo de la DM2 tienen limitaciones en cuanto a la capacidad de recuerdo y la deseabilidad social de las respuestas. Ello se refleja en la alta adherencia a medicamentos reportada por ambos grupos en la medición basal, que es muy superior a las reconocidas por la literatura, en general inferiores a $50 \%{ }^{26}$. En cambio, a través de las consejerías telefónicas, se obtuvo información más fidedigna respecto a dificultades enfrentadas por los pacientes para adherir al tratamiento, algunas de las cuales pudieron ser resueltas conjuntamente por el paciente y la enfermera de tele-cuidado (desconocimiento del paciente de la indicación correcta, problemas relacionados con acceso oportuno a receta médica, stock disponible en farmacia). Este efecto podría ser la principal explicación para la estabilización de la HbAlc en el GI.

Si bien la pérdida de sujetos entre la medición inicial y la final fue importante, ella estuvo considerada en el cálculo del tamaño muestral y, por lo tanto, no tuvo impacto en la validez estadística de los resultados. El número de sujetos estudiados y el período de seguimiento eran insuficientes para demostrar una disminución de las hospitalizaciones en el grupo intervenido.

En conclusión, los resultados muestran que el modelo ATAS, articulado con la atención habitual 
que ofrece el PSCV es eficaz en limitar el incremento de la $\mathrm{HbA} 1 \mathrm{c}$ en pacientes con DM2, mejorar su asistencia a los controles de salud periódicos, reducir las consultas de urgencia y desarrollar en el paciente un positivo sentido de autoeficacia en el control de su enfermedad crónica.

El MINSAL, a través del servicio telefónico "Salud Responde", permite a los pacientes recibir orientación profesional en el manejo de síntomas y utilización de los servicios de salud, 24 horas al día, 7 días a la semana ${ }^{33}$. Sería interesante replicar este estudio integrando el modelo ATAS con "Salud Responde".

Agradecimientos: Al Dr. Antonio Arteaga, por su asesoría en la interpretación clínica de los resultados. $\mathrm{Al}$ ingeniero Iñigo Meza por su apoyo en el área informática. A los Dres. Sibila Iñiguez, Sergio Araya, la Nutricionista Francisca Zúñiga y los profesionales de los Centros de Salud Bernardo Leighton y San Gerónimo, de la Corporación Municipal de Puente Alto. A los pacientes y sus familias, sin los cuales habría sido imposible realizar este estudio.

\section{Referencias}

1. Asociación de Diabéticos de Chile. Estudio Nacional de Prevalencia de Diabetes en Chile 2006. Disponible en: http://www.adich.cl/adich4.php?page=Estudio [Consultado el 13 de julio de 2009].

2. Ministerio de Salud de Chile, Pontificia Universidad Católica de Chile. Resultados I Encuesta de Salud, Chile 2003. Disponible en: http://epi.minsal.cl/epi/html/invest/ENS/folletoENS.pdf [Consultado el 14 de julio de 2009].

3. Wagner E, Austin B, Vonkorff M. Improving outcomes in chronic illness. Managed Care Quarterly 1996; 4: 12-25.

4. Hart W, Collazo M. Costos del diagnóstico y tratamiento de la diabetes mellitus en diferentes países del mundo. Rev Cubana Endocrinol 1998; 9:212-20.

5. Piette J, Lange I, Issel M, Campos S, Bustamante C, Sapag J, et al. Use of telephone care in a cardiovascular disease management program for type 2 diabetes patients in Santiago, Chile. Chronic Illness 2006; 2: 87-96.

6. Weinberger M, Kirkman MS, Samsa GP, Shortliffe EA, Landsman PB, Cowper PA, et al. A nurse-coordinated intervention for primary care patients with non-insulindependent diabetes mellitus: impact on glycemic control and health- related quality of life. J Gen Intern Med
1995; 10: 59-66.

7. Aubert R, Herman W, Waters J, Moore W, Sutton D, Peterson $\mathrm{B}$, et al. Nurse Case Management to Improve Glycemic Control in Diabetic Patients in a Health Maintenance Organization. Ann Intern Med 1998; 129: 605-12.

8. Piette J, Weinberger M, Kraemer F, McPhee S. Impact of Automated Calls With Nurse Follow-Up on Diabetes Treatment Outcomes in a Department of Veterans Affairs Health Care System. Diabetes Care 2001; 24: 202-8.

9. Lattimer V, George S, Thompson F, Thomas E, Mullee $\mathrm{M}$, Turnbull J, et al. Safety and effectiveness of nurse telephone consultation in out of hours primary care: randomized controlled trial. BMJ 1998; 317: 1054-9.

10. Barr Taylor C, Houston Miller N, Reilly K, Greenwald G, Cunning D, Deeter A, et al. Evaluation of a Nurse Care Management System to Improve Outcomes in Patients with Complicated Diabetes. Diabetes Care 2003; 26: 1058-63.

11. Stacey D, Noorani H, Fisher A, Robinson D, Joyce J, Pong R. Telephone Triage Service: Systematic Review and a Survey of Canadian Call Centre Programs. Ottawa Canadien Coordinating Office for Health Technology Assessment 2003. Technology report no 43. Disponible en http://www.cadth.ca/media/pdf/180_teletriage_tr_e. pdf [Consultado el 14 de julio 2009].

12. Toobert D, Hampson S, Glasgow R. The Summary of Diabetes Self-Care Activities Measure. Diabetes Care 2000; 23: 943-50.

13. Stanford Patient Education Research Center. Spanish Social/Role Activities Limitations Scale. s/f (Disponible en: http://patienteducation.stanford.edu/research/activitieslimitesp.html [Consultado el 14 de julio 2009]

14. Stanford Patient Education Research Center. Spanish Diabetes Self-efficacy s/f Disponible en: http://patienteducation.stanford.edu/research/sediabetesesp.html. [Consultado el 14 de julio 2009]

15. Ministerio de Salud. Guía Clínica Hipertensión Arterial Primaria o Esencial en personas de 15 años y más. 2006; 1ra Ed.Santiago. Minsal.

16. Hedeker D, Gibbons R. Longitudinal Data Analysis. John Wiley and Sons, Inc. New Jersey, 2006.

17. R Development Core Team. R: A Language and Environment for Statistical Computing. R Foundation for Statistical Computing. 2008 Vienna, Austria. Disponible en: http://www.R-project.org [Consultado el 1 de junio de 2009].

18. Pinheiro, JC, Bates, DM. Mixed-effects models in S and S-PLUS. Springer 2000.

19. Intensive Blood-Glucose Control with Sulphonylureas or Insulin Comparaed With Conventional Treatment and 
Risk of Complications in Patients with Type 2 Diabetes (UKPDS 33). UK Prospective Diabetes Study (UKPDS) Group. Lancet 1988; 352: 837-53.

20. Yudkin JS. The United Kingdom Prospective Diabetes Study- everything you need to know about diabetes but were afraid to ask? Eur Heart J 1999; 20: 781-83.

21. Resnick B. Review: Diabetes Management: The Hidden Challenge of Managing Hyperglycemia in Long-Term Care Settings. Annals of Long-Term Care. Disponible en www.annalsoflongtermcare.com/article/4499 [Consultado el 4 de agosto 2008]

22. Khaw K, Wareham N, Bingham S, Luben R, Welch A, Day N. Association of Hemoglobin Alc with Cardiovascular Disease and Mortality in Adults: The European Prospective Investigation into Cancer in Norfolk. Ann Intern Med 2004; 141: 413-21.

23. Ministerio de Salud de Chile. Programa de Salud Cardiovascular: Reorientación de los Programas de Hipertensión y Diabetes. 2002. Disponible en: http://www.minsal. cl/ici/padulto/doc/Reorientacion\%20de\%20los\%20 Programas\%20de\%20Hipertension\%20y\%20Diabetes. pdf [Consultado el 14 de julio 2009].

24. Karter AJ, Parker MM, Moffet HH, Ahmed AT, Ferrara A, Llu JY, et al. Missed appointment and poor glycemic control: an opportunity to identify high risk diabetic patients. Med Care 2004; 42: 110-5.

25. Schechtman J, Schorling J, Voss J. Appointment Adherence and Disparities in Outcomes Among Patients with
Diabetes. J GIM 2008; 23: 1685-87.

26. OMS. Adherencia a los tratamientos a largo plazo. Pruebas para la acción. 2004. Disponible en: http:// www.paho.org/Spanish/AD/DPC/NC/nc-adherencia. pdf [Consultado el 3 agosto 2008].

27. Ingaramo R, Vita $\mathrm{N}$, Bendersky $\mathrm{M}$, Arnolt $\mathrm{M}$, Bellido C, Piskorz D, et al. Estudio Nacional Sobre Adherencia al Tratamiento (ENSAT). Rev Fed Arg Cardiol 2005; 34 : 104-11.

28. Evans E. Compliance with Antihypertensive Medication. Can Fam Physician 1985; 31:335-40.

29. Gil V, Pineda M, Martínez J, Belda J., Santos Ml, Merino J. Validez de 6 métodos indirectos para valorar el cumplimiento terapéutico en hipertensión arterial. Med Clin (Barc) 1994; 102: 532-36.

30. Trief P, Teresi J, Izquierdo R, Morin P, Goland R, Field L, et al. Psychosocial Outcomes of Telemedicine Case Management for Elderly Patients With Diabetes. Diabetes Care 2007; 30: 1266-68.

31. Trief P, Sandberg J, Izquierdo R, Morin P, Shea S, Brittain $\mathrm{R}$, et al. Diabetes Management Assisted by Telemedicine: Patient Perspectives. Telemedicine and e-Health 2007; 14: 647-55.

32. Bandura A. Self-Efficacy: The Exercise of Control. New York. Freeman and Company; 2000.

33. Ministerio de Salud. Servicio Telefónico "Salud Responde" Disponible en: http://www.saludresponde.cl [Consultado el 13 de julio 2009]. 